\title{
Surface acoustic wave velocity and elastic constants of cubic GaN
}

\author{
Rafael J. Jiménez Riobóo, ${ }^{1}$ Ramon Cuscó, ${ }^{2}$ Carlos \\ Prieto, ${ }^{1}$ Sergei V. Novikov, ${ }^{3}$ and Lluís Artús ${ }^{4}$ \\ ${ }^{1}$ Instituto de Ciencia de Materiales de Madrid (CSIC), \\ Sor Juana Inés de la Cruz 3, E-28049 Madrid, Spain \\ ${ }^{2}$ Institut Jaume Almera (ICTJA), Consell Superior d'Investigacions Cientifiques (CSIC), \\ Lluís Solé i Sabarís s.n., E-08028 Barcelona, Spain \\ ${ }^{3}$ School of Physics and Astronomy, \\ University of Nottingham, Nottingham NG7 2RD, UK \\ ${ }^{4}$ Institut Jaume Almera (ICTJA), Consell Superior d'Investigacions Cientifiques (CSIC), \\ Lluís Solé i Sabarís s.n., E-08028 Barcelona, Spain
}

\begin{abstract}
We present high resolution Brillouin scattering measurements on cubic GaN layers grown on GaAs substrate. By using a suitable scattering geometry, scattering by surface acoustic waves was recorded for different azimuthal angles and the surface acoustic wave velocities were determined. The comparison of the experimental results with numerical simulations of the azimuthal dependence of the surface wave velocity shows good agreement and allowed us to determine a consistent set of elastic constants for $c-\mathrm{GaN}$.
\end{abstract}


In recent years, the development of solid-state light-emitter technology has attracted much research effort into the group III nitrides. The InGaN-GaN material system has produced efficient light emitting diodes in the yellow, green and blue regions and form the basis of current solid-state lighting technology for white lighting applications. ${ }^{1}$ On the other hand, the unique properties of GaN (wide bandgap, high breakdown electric field, high electron mobility) make this material a promising candidate for the next generation of microwave power electronics ${ }^{2}$ and high temperature, high frequency devices. ${ }^{3}$ The group III nitrides normally crystallize in the stable, hexagonal, wurtzite structure. For conventional growth of quantum wells along the polar $c$ axis, strong built-in electric fields caused by gradients of spontaneous and piezoelectric polarization develop which cause the charge separation of electron-hole pairs (quantum Stark effect). This results in the redshift of the emission wavelength and in a reduced emission efficiency. ${ }^{1,4}$ Growth on non-polar or semi-polar faces of hexagonal GaN is being explored to overcome this problem. ${ }^{4,5}$ An alternative route consists in using the metastable zinc-blende polytype of GaN which is free of built-in electric fields in the typical (100) growth direction..$^{6,7}$

The growth of non-polar (100) oriented cubic (zincblende) GaN layers can be achieved by molecular beam epitaxy (MBE) on (100) cubic substrates under specific growth conditions. ${ }^{8}$ Despite the increasing technological interest in zincblende GaN, this polytype has received so far much less attention than its wurtzite counterpart and their physical properties are not well known yet. Only recently a comprehensive study of the optical properties of cubic GaN in the near-band-gap excitonic region has been published. ${ }^{9}$ More recently, the anharmonic phonon decay in c-GaN has been investigated by means of Raman scattering. ${ }^{10}$ Whereas Brillouin scattering measurements providing a reliable experimental determinaton of the elastics constants were reported in wurtzite GaN, ${ }^{11,12}$ the present knowledge of the elastic constants of cubic GaN comes mainly from molecular-dynamics and densityfunctional-theory calculations. ${ }^{13-18}$ To our knowledge, only one of the three independent elastic constants of c-GaN has been experimentally determined by means of picosecond acoustic experiments. ${ }^{19}$

In this Letter, we present a high resolution Brillouin scattering (HRBS) study of the elastic constants of cubic GaN. Our measurements were performed on a high-quality zincblende GaN layer grown on a semi-insulating (100) GaAs substrate by plasma-assisted molecular beam epitaxy (PA-MBE) in a MOD-GENII system using $\mathrm{As}_{2}$ as a surfactant to initiate the 


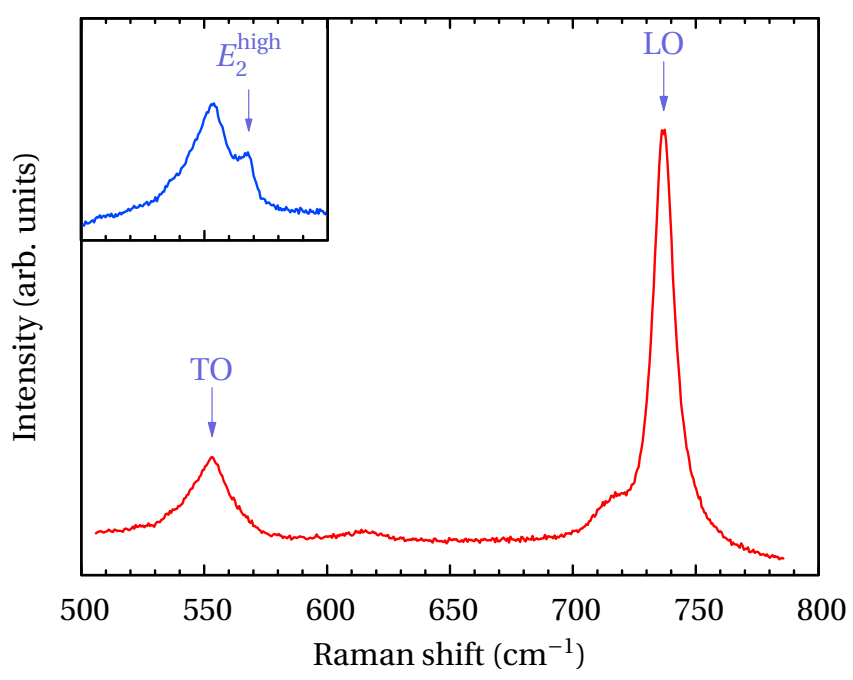

FIG. 1: Raman spectrum in $z(x y) \bar{z}$ backscattering configuration of the cubic GaN layer studied. Inset, Raman spectrum of a different cubic layer with higher hexagonal phase content.

growth of the cubic phase material. Details of the growth procedure are given elsewhere. ${ }^{8}$ Raman scattering measurements were used to assess the cubic phase purity of the sample. The Raman spectra were excited with the $532 \mathrm{~nm}$ line of a diode-pumped Nd:YAG solidstate laser and were analyzed using a Jobin-Yvon T64000 spectrometer. The thickness of the layer was determined to be $h=515 \pm 16 \mathrm{~nm}$ by means of spectroscopic ellipsometry using a SOPRA GES5E system and the Winelli II software. The Brillouin measurements were carried out using a 2060 Beamlock Spectra Physics $\mathrm{Ar}^{+}$laser fitted with an intracavity temperature-stabilized, single-mode and single-frequency z-lok etalon $\left(\lambda_{0}=514.5 \mathrm{~nm}\right)$ as a light source. The scattered light was analyzed using a Sandercock-type $3+3$ tandem Fabry-Pérot interferometer. ${ }^{20}$ A more detailed description of the experimental set-up is given elsewhere. ${ }^{21}$ Typical values for finesse and constrast were 150 and $10^{9}$, respectively.

To assess the crystalline quality and phase purity of the studied cubic GaN layer, preliminary Raman scattering measurements were performed. Figure 1 displays the Raman spectrum in $z(x y) \bar{z}$ backscattering configuration of the cubic GaN layer used in this study. The spectrum is dominated by the symmetry allowed LO mode at $737 \mathrm{~cm}^{-1}$. The measured full width at half maximum of the LO peak $\left(9.9 \mathrm{~cm}^{-1}\right)$ compares well with cubic GaN Raman spectra published in the literature. ${ }^{10,22}$ The low-frequency shoulder observed at 714 $\mathrm{cm}^{-1}$ was assigned to a second-order $\mathrm{TA}(L)+\mathrm{TO}(L)$ combination mode. ${ }^{10}$ The weaker peak 


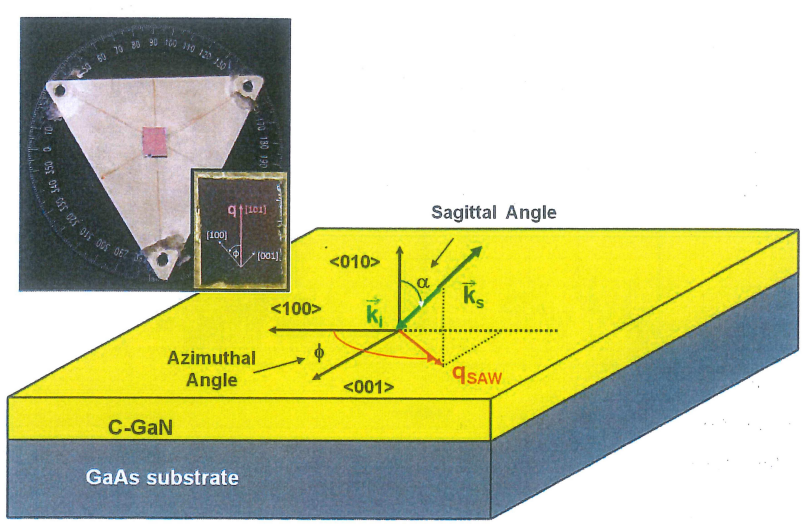

FIG. 2: Schematic representation of the surface Brillouin scattering geometry used in the measurements. The incident light of wave vector $\boldsymbol{k}_{i}$ is at an angle $\alpha$ (saggital angle) from the surface normal. The backscattered light is collected at $180^{\circ}$ with wave vector $\boldsymbol{k}_{s}$. The light is polarized in the scattering plane and the scattered phonon wave vector is $\boldsymbol{q}_{\mathrm{SAW}}$. The scattering plane is rotated about the $\langle 010\rangle$ axis by an angle $\phi$ (azimuthal angle). Inset, a view of the cubic GaN sample mounted on the sample holder.

observed at $543 \mathrm{~cm}^{-1}$ corresponds to the TO mode of the cubic GaN. Although this mode is forbidden in backscattering from $\{100\}$ faces, it is detected in the Raman spectra due to the forward scattering contribution of the light reflected at the interface between the transparent layer and the substrate. ${ }^{10,23,24}$ No signal from the $E_{2}^{\text {high }}$ mode of the wurtzite GaN can be observed in the spectra. Given the sizable intensity of the $E_{2}^{\text {high }}$ peak characteristic of the hexagonal phase, its absence in the Raman spectrum indicates the high phase purity of the cubic GaN layer used in this study. For comparison, in the inset of Fig. 1 we display a Raman spectrum of a cubic GaN layer which contains also some hexagonal phase. In this sample, the emergence of a distinct, weak peak at $568 \mathrm{~cm}^{-1}$ corresponding to the $E_{2}^{\text {high }}$ mode of the wurtzite GaN can be clearly observed. These spectra demonstrate the high sensitivity of Raman scattering for characterizing the phase purity of cubic GaN layers.

To obtain information about the elastic constants of cubic GaN, the surface acoustic wave velocity $\left(v_{\text {SAW }}\right)$ was determined from HRBS measurements. Even though Brillouin scattering in transparent materials is dominated by the elasto-optic scattering by bulk modes, the observation of excitations localized near the surface (i.e. Raighley surface waves) is possible 


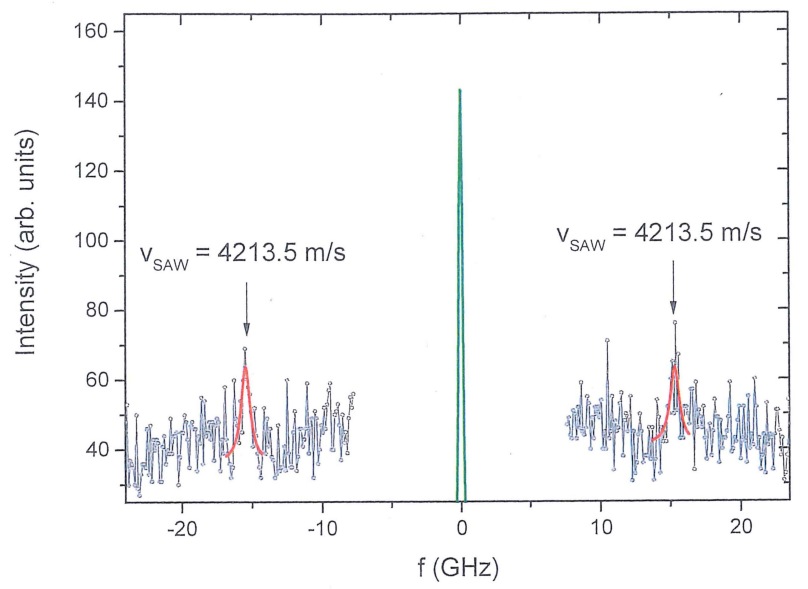

FIG. 3: HRSBS spectrum of the cubic GaN sample for $\alpha=70^{\circ}$ in the [101] direction. Lorentzian line shapes have been fitted to the Rayleigh peaks to accurately obtain the spectral frequency shift of the light corresponding to scattering with the surface acoustic phonon mode.

in transparent films deposited on an opaque substrate. ${ }^{25}$ In order to couple the incident light beam to the surface phonons, the incident beam was polarized in the scattering plane. The backscattered light was collected at $180^{\circ}$ according to the scattering geometry shown in Fig. 2. In this backscattering geometry the SAW wave vector $\boldsymbol{q}_{\mathrm{SAW}}$ is parallel to the sample surface and its magnitude is given by $q_{\mathrm{SAW}}=4 \pi \sin \alpha / \lambda_{0}$, where $\lambda_{0}$ is the laser wavelength in vacuum and $\alpha$ is the sagittal angle. The SAW propagation velocity can then be obtained from the surface phonon frequency $\nu_{\mathrm{SAW}}$ as

$$
v_{\mathrm{SAW}}=\frac{\nu_{\mathrm{SAW}} \lambda_{0}}{2 \sin \alpha}
$$

The scattering angle used in the measurements $\left(\alpha=70^{\circ}\right)$ provides a $q_{\mathrm{SAW}} h$ value of 11.8 , which is high enough for the Rayleigh modes to be in the asymptotic region where the influence of the substrate is negligible. ${ }^{26}$ Figure 3 displays a typical HRSBS spectrum obtained for $\alpha=70^{\circ}$ and $\phi=45^{\circ}$ from the cubic GaN layer. A Lorentzian line-shape fit to the Rayleigh peak yields an accurate value of the SAW propagation velocity via Eq. (1).

Crystals with cubic symmetry exhibit four-fold symmetry in the $v_{\text {SAW }}$ values within the (010) plane. By rotating the sample about the surface normal, the azimuthal angle can be varied and thus the direction of scattering wave vector $\boldsymbol{q}_{\mathrm{SAW}}$ can be selected within the (010) plane. For the HRSBS experiments, the sample was placed on a sample holder that was attached to a goniometer, so that it was possible to accurately fix the sagittal angle $\alpha$ and 
rotate the sample about the surface normal to change the azimuthal angle $\phi$, as can be seen in Fig. 2. In this way, the SAW propagation velocity in different crystallographic directions within the sample plane can be experimentally probed by HRSBS measurements.

Since the SAW propagation velocity is determined by the elastic constants of the material, we can extract information about the three independent elastic constants of the cubic layer from our HRSBS measurements.

For a crystal with cubic symmetry, the Rayleigh SAW velocities in the [100] and [101] directions are determined, respectively, by the secular equations ${ }^{27,28}$

$$
\rho v_{\mathrm{SAW}}^{2}-\left(\frac{c_{11}}{c_{44}} \times \frac{c_{44}-\rho v_{\mathrm{SAW}}^{2}}{c_{11}-\rho v_{\mathrm{SAW}}^{2}}\right)^{\frac{1}{2}}\left(c_{11}-\frac{c_{12}^{2}}{c_{11}}-\rho v_{\mathrm{SAW}}^{2}\right)=0
$$

and

$$
\rho v_{\mathrm{SAW}}^{2}-\left(\frac{c_{11}}{c_{44}} \times \frac{c_{44}-\rho v_{\mathrm{SAW}}^{2}}{\frac{1}{2}\left(c_{11}+c_{12}+2 c_{44}\right)-\rho v_{\mathrm{SAW}}^{2}}\right)^{\frac{1}{2}}\left(\frac{1}{2}\left(c_{11}+c_{12}+2 c_{44}\right)-\frac{c_{12}^{2}}{c_{11}}-\rho v_{\mathrm{SAW}}^{2}\right)=0 .
$$

Here $\rho=6.103 \mathrm{~g} \mathrm{~cm}^{-3}$ is the mass density of cubic GaN estimated from the lattice parameter given in Ref. 29 and $c_{11}, c_{12}$, and $c_{44}$ are the three independent elastic constants of cubic GaN. From the HRSBS measurements we have determined $v_{\mathrm{SAW}}^{[100]}=3866.9 \mathrm{~m} \mathrm{~s}^{-1}$ and $v_{\text {SAW }}^{[101]}=4220.4 \mathrm{~m} \mathrm{~s}^{-1}$. Then, Eqs. (2) and (3) constitute an undetermined system of two equations with three unknowns. To reduce the number of unknowns and render the system determinate, we consider the longitudinal sound velocity and the corresponding elastic constant $c_{11}$ experimentally measured in cubic GaN by picosecond acoustic measurements at $4.7 \mathrm{~K} .{ }^{19}$ The sound velocity measured in wurtzite-type GaN was found to be independent of temperature within experimental error, ${ }^{19}$ so it is reasonable to consider the $c_{11}$ value of $285 \mathrm{GPa}$ determined at low temperature in our room temperature analysis. By solving Eqs. (2) and (3) with $\rho=6.103 \mathrm{~g} \mathrm{~cm}^{-3}$ and $c_{11}=285 \mathrm{GPa}$ as input parameters, we can determine the elastic constants $c_{12}$ and $c_{44}$ from the measured SAW velocities. In Table I, the values we obtain are compared with theoretical values derived from a number of computational approaches such as density functional theory, molecular dynamics, and Keating model available in the literature.

Although the different calculational approaches yield a certain dispersion in the elastic constant values, it is worth noticing that the set of $c_{i j}$ values determined from experimental 
TABLE I: Elastic constants for c-GaN as determined in this work from HRBS measurements of the SAW compared with values predicted by theoretical calculations.

\begin{tabular}{cccc}
\hline \hline$c_{11}(\mathrm{GPa})$ & $c_{12}(\mathrm{GPa})$ & $c_{44}(\mathrm{GPa})$ & Ref. \\
\hline $285 \pm 8^{\mathrm{a}}$ & $149 \pm 9^{\mathrm{b}}$ & $157.7 \pm 0.6^{\mathrm{b}}$ & \\
264 & 153 & 68 & 13 \\
296 & 154 & 206 & 14 \\
293 & 159 & 155 & 15 \\
274.2 & 166.1 & 199 & 16 \\
295 & 155.1 & 189.4 & 17 \\
255 & 133 & 177 & 18 \\
305 & 128 & 147 & 30 \\
325 & 142 & 147 & 31 \\
\hline \hline
\end{tabular}

${ }^{a}$ Experimental value taken from Ref. 19

b This work: experimental values derived from HRBS measurements.

measurements of the Rayleigh SAW velocities in the present work are consistent with the predicted values and display relative deviations less than $2 \%$ in relation to the averaged theoretical values given in Table I.

In order to check the validity of the obtained results, the set of elastic constants have been used to simulate the scattered light intensity using the Green's function formalism introduced by Zhang et $a l .{ }^{32}$ The dependence of the SAW velocity on the azimuthal angle for the c-GaN/GaAs sample studied is plotted in Fig. 4. An excellent agreement is found between the calculated azimuthal dependence and the SAW velocity measured by HRBS experiments along different directions, which confirms the consistency of the set of elastic constants determined in this work.

In summary, we have carried out HRBS measurements to determine the SAW velocity in a (010)-oriented c-GaN layer for the [100] and [101] propagation directions. From the analysis of the Rayleigh SAW peak frequency a consistent set of elastic constants $c_{11}, c_{12}$ and $c_{44}$ has been experimentally determined for the first time in c-GaN. The obtained values agree well with the mean values of the theoretical predictions found in the literature. The 


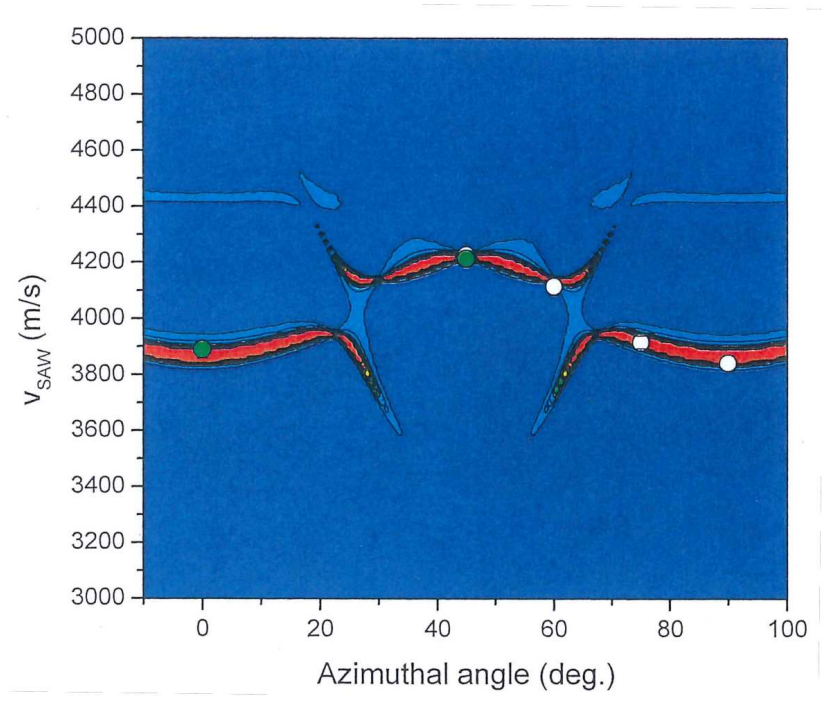

FIG. 4: Simulation of the azimuthal angle dependence of the SAW velocity for the c-GaN layer on GaAs using the set of elastic constants determined in this work (see Table I). The circles represent SAW velocities at different azimuthal angles determined by HRBS measurements.

azimuthal dependence of the SAW velocity found in the HRBS experiments can be accurately simulated using the obtained set of elastic constants.

\section{Acknowledgments}

This work was partially supported by the Spanish MINECO under project MAT201237276-C03-01 and from Comunidad de Madrid project S2013/MIT-2740 (PHAMA_2.0-CM).

1 M. R. Krames, O. B. Shchekin, R. Mueller-Mach, G. O. Mueller, L. Zhou, G. Harbers, and M. G. Craford, J. Disp. Technol. 3, 160 (2007).

2 U. Mishra, S. Likun, T. Kazior, and Y.-F. Wu, Proc. IEEE 96, 287 (2008).

3 M. Manfra, N. Weimann, Y. Baeyens, P. Roux, and D. Tennant, Electron. Lett. 39, 694 (2003).

4 S. Pleasants, Nat. Photonics 7, 585 (2013).

5 B. A. Haskell, F. Wu, S. Matsuda, M. D. Craven, P. T. Fini, S. P. DenBaars, J. S. Speck, and S. Nakamura, Appl. Phys. Lett. 83, 1554 (2003).

6 S. Li, J. Schörmann, D. J. As, and K. Lischka, Appl. Phys. Lett. 90, 071903 (2007). 
7 C. J. M. Stark, T. Detchprohm, S. C. Lee, Y.-B. Jiang, S. R. J. Brueck, and C. Wetzel, Appl. Phys. Lett. 103, 232107 (2013).

8 S. V. Novikov, N. Zainal, A. V. Akimov, C. R. Staddon, A. J. Kent, and C. T. Foxon, J. Vac. Sci. Technol. B 28, C3B1 (2010).

9 M. Feneberg, M. Röppischer, C. Cobet, N. Esser, J. Schörmann, T. Schupp, D. J. As, F. Hörich, J. Bläsing, A. Krost, et al., Phys. Rev. B 85, 155207 (2012).

10 R. Cuscó, N. Domènech-Amador, S. Novikov, C. T. Foxon, and L. Artús, Phys. Rev. B 92, $075206(2015)$.

11 A. Polian, M. Grimsditch, and I. Grzegory, J. Appl. Phys. 79, 3343 (1996).

12 M. Yamaguchi, T. Yagi, T. Azuhata, T. Sota, K. Suzuki, S. Chichibu, and S. Nakamura, J. Phys. Cond. Mat. 9, 241 (1997).

13 M. E. Sherwin and T. J. Drummond, J. Appl. Phys. 69, 8423 (1991).

14 K. Kim, W. R. L. Lambrecht, and B. Segall, Phys. Rev. B 50, 1502 (1994).

15 A. F. Wright, J. Appl. Phys. 82, 2833 (1997).

16 K. Shimada, T. Sota, and K. Suzuki, J. Appl. Phys. 84, 4951 (1998).

17 F. Benkabou, H. Aourag, P. J. Becker, and M. Certier, Mol. Simul. 23, 327 (2000).

18 Z. Usman, C. Cao, G. Nabi, D. Y. Kun, W. S. Khan, T. Mehmood, and S. Hussain, J. Phys. Chem. A 115, 6622 (2011).

19 D. Moss, A. V. Akimov, S. V. Novikov, R. P. Campion, C. R. Staddon, N. Zainal, C. T. Foxon, and A. J. Kent, J. Phys. D: Appl. Phys. 42, 115412 (2009).

20 J. R. Sandercock, in Light Scattering in Solids III, Topics in Applied Physics (Springer-Verlag, Berlin, 1982), vol. 51, pp. 173-206.

21 R. J. Jiménez-Riobóo, M. García-Hernández, C. Prieto, J. J. Fuentes-Gallego, E. Blanco, and M. Ramírez-del Solar, J. Appl. Phys. 81, 7739 (1997).

22 G. Mirjalili, T. Parker, S. Farjami Shayesteh, M. Bülbül, S. Smith, T. Cheng, and C. Foxon, Phys. Rev. B 57, 4656 (1998).

23 H. Siegle, L. Eckey, A. Hoffmann, C. Thomsen, B. Meyer, D. Schikora, M. Hankeln, and K. Lischka, Solid State Comm. 96, 943 (1995).

24 R. Cuscó, N. Domènech-Amador, T. Hatakeyama, T. Yamaguchi, T. Honda, and L. Artús, J. Appl. Phys. 117, 185706 (2015).

25 V. Bortolani, F. Nizzoli, G. Santoro, and J. R. Sandercock, Phys. Rev. B 25, 3442 (1982). 
${ }^{26}$ R. J. Jiménez-Riobóo, R. Cuscó, R. Oliva, N. Domènech-Amador, C. Prieto, J. Ibáñez, C. Boney, A. Bensaoula, and L. Artús, Appl. Phys. Lett. 101, 062103 (2012).

27 A. G. Every, L. M. Kotane, and J. D. Comins, Phys Rev B 81 (2010).

28 L. M. Kotane, J. D. Comins, A. G. Every, and J. R. Botha, J. Phys. Conf. Ser. 278, 012001 (2011).

29 I. Vurgaftman and J. R. Meyer, Journal of Applied Physics 94, 3675 (2003).

30 A. A. Marmalyuk, R. K. Akchurin, and V. A. Groblyev, Inorganic Materials 34, 691 (1998).

31 S. Y. Davydov and A. V. Solomonov, Tech. Phys. Lett. 25, 601 (1999).

32 X. Zhang, J. D. Comins, A. G. Every, P. R. Stoddart, W. Pang, and T. E. Derry, Phys. Rev. B 58, $13677(1998)$. 